\title{
Carbon Nanotube Sensing Skins for Spatial Strain and Impact Damage Identification
}

\author{
Kenneth J. Loh • Tsung-Chin Hou • Jerome P. Lynch • \\ Nicholas A. Kotov
}

Received: 28 April 2008 / Accepted: 14 February 2009 / Published online: 19 March 2009

(C) Springer Science+Business Media, LLC 2009

\begin{abstract}
Impact damage, excessive loading, and corrosion have been identified as critical and long-term problems that constantly threaten the integrity and reliability of structural systems (e.g., civil infrastructures, aircrafts, and naval vessels). While a variety of sensing transducers have been proposed for structural health monitoring, most sensors only offer measurement of structural behavior at discrete structural locations. Here, a conformable carbon nanotubepolyelectrolyte sensing skin fabricated via the layer-by-layer technique is proposed to monitor strain and impact damage over spatial areas. Specifically, electrical impedance tomographical (EIT) conductivity mapping techniques are employed to offer two-dimensional damage maps from which damage location and severity can be easily and accurately quantified. This study deposits carbon nanotube-based sensing skins upon metallic structural plates with electrodes installed along the plate boundary. Based on boundary electrical measurements, EIT mapping captures both strain in the underlying substrate as well as damage (e.g., permanent
\end{abstract}

K.J. Loh

Department of Civil and Environmental Engineering, University of California, One Shields Avenue, 3155 Engineering III, Davis, CA 95616, USA

e-mail: kjloh@ucdavis.edu

T.-C. Hou · J.P. Lynch $(\bowtie)$

Department of Civil and Environmental Engineering, University of Michigan, 2350 Hayward Street, Ann Arbor, MI 48109-2125, USA

e-mail: jerlynch@umich.edu

\section{N.A. Kotov}

Department of Chemical Engineering, University of Michigan, 2300 Hayward Street, Ann Arbor, MI 48109-2125, USA

e-mail: kotov@umich.edu deformation and cracking) introduced using an impact apparatus.

Keywords Carbon nanotube nanocomposite . Electrical impedance tomography · Impact damage detection .

Layer-by-layer $\cdot$ Sensing skin $\cdot$ Structural health monitoring

\section{Introduction}

The challenges associated with managing aging civil infrastructures, aerospace, and mechanical engineered systems demand new sensing technologies to ensure long-term safety and reliability. In addition, harsh operational and environmental conditions coupled with the lack of robust monitoring and maintenance schemes often accelerate structural degradation. In extreme cases, undetected structural damage such as impact damage, corrosion, and fatigue can lead to catastrophic system failure. For instance, undiagnosed corrosion of aircraft fuselages can result in over-stressing of rivets and stress-corrosion crack formation; an example of what can occur if damage is left undetected is the Aloha Airlines plane which ripped apart in-flight in 1988 [1]. More recently, impact damage compromised the integrity of the thermal insulation heat shield of the Columbia Space Shuttle which exploded during re-entry [2]. In addition to such fatal catastrophic structural failures, an abundance of economic resources are dedicated to inspecting and repairing existing structures. According to Njord and Meyer [3], more than $\$ 91$ billion is spent annually to maintain the U.S. inventory of highways and bridges. While this cost may seem significant, it is estimated that at least an additional \$128 billion is required to upgrade existing structures to meet current standards. 
As a means to mitigate the economic burden of structural maintenance and the risk of catastrophic failures, the field of structural health monitoring (SHM) offers a diverse suite of sensing and algorithmic technologies that identify structural degradation so as to facilitate timely repairs. While many SHM techniques have been proposed, the majority rely on correlating changes in global structural properties to damage [4]. In order to detect component-level damage, techniques such as global modal analysis are often not sufficiently sensitive to local damage (e.g., fatigue cracks, steel reinforcement corrosion, among others) [4]. While strain gages can be installed to obtain local structural measurements, their main disadvantage is that they can only provide response data at one discrete location in the structure. Accurate damage detection requires either a dense network of these point-sensors or accurate models for extrapolating localized strain fields to the remainder of the structure.

As opposed to relying on global-based SHM methods, guided-waves show tremendous promise for damage detection and localization in thin metallic structures [5, 6]. With ultrasonic waves able to propagate over long distances, this sensing approach provides the sensor with a large interrogation zone. For instance, Giurgiutiu, et al. [7] bonds multiple arrays of surface-mounted piezoelectric transducers onto aluminum alloy aircraft panels for generating Lamb waves to detect cracks and corrosion damage. Using piezoelectric sensor/actuator responses, reflected echo signals are employed for accurate detection of damage due to hairline slits, seeded cracks, and impact. Similarly, Park, et al. [8] has validated piezoelectric patches for identifying cracks developed in welded zones of 1/8-scale bridge truss members. Simulated damage by loose bolts and induced cracks are successfully detected via a Lamb-wave approach combined with a prescribed damage threshold index. On the other hand, when combining distributed piezoelectric sensors/actuators with a wavelet-based data processing technique, Sohn, et al. [9] identifies the location of delamination within large $61 \times 61 \mathrm{~cm}^{2}$ composite plates. While the aforementioned guided-wave techniques are fairly robust for identifying damage in simple structural components, computational demand increases dramatically, and accuracy decreases when structural geometries are complex and when multiple damage sites are within the active sensors' interrogation zone. In addition, a dense network of piezoelectric sensors and actuators, combined with computationallyintensive algorithms, are still required for detecting small damage features. Moreover, application of guided-waves on complex structures generally requires a priori geometrical knowledge of the undamaged structure to distinguish structural features from detected damage [5], a problem that is exacerbated by temperature variations.

More recently, advances in nanotechnology have brought forth new materials and fabrication tools for developing high-sensitivity thin film sensors that are suitable for use in SHM [10]. In fact, carbon fullerenes and nanotubes [11] show tremendous potential for use in multifunctional materials. For example, it has been shown that single-walled carbon nanotubes (SWNT) possess a Young's Modulus of approximately $1 \mathrm{TPa}$ and exhibit near-ballistic transport-type electronic conductivities [12]. The strength characteristics of SWNTs have led to many exploring their use for reinforcing polymeric and ceramic materials [13-15]. While mechanical durability is one desirable attribute of carbon nanotube composites, another is the embedment of sensing functionalities. Specifically, the large surface area and high aspect ratio of SWNTs are ideally suited for chemical functionalization; binding molecules to the surface of SWNTs allow electromechanical and electrochemical sensing transduction mechanisms to be encoded into a carbon nanotube composite.

In this study, SWNTs are employed as a building block for the design and fabrication of multifunctional "sensing skins" that are capable of monitoring structural damage without having to probe multiple discrete sensor locations to infer the characteristics (i.e., type, location, and severity) of damage. The proposed sensing skin seeks to: 1) spatially image deformations (strain) and 2) identify and locate breaks in the skin that could be attributed to cracking of the underlying structure or due to physical impacts on the skin. Using a layer-by-layer (LbL) self-assembly process, individual SWNTs are functionalized with various polyelectrolyte species for embedding electromechanical sensing transduction mechanisms within a composite's morphology for strain sensing $[16,17]$. Realization of a sensing skin with spatial resolution is achieved by coupling the nanocomposites with an electrical impedance tomographic (EIT) spatial conductivity imaging technique [18-21]. In short, the EIT conductivity mapping technique relies on repeated electrical measurements at the film boundary to inversely reconstruct the skin's spatial conductivity distribution. This paper begins with a brief introduction to electrical impedance tomography. Then, the layer-by-layer skin fabrication process is described in detail. Next, the carbon nanotube sensing skins are experimentally validated to detect tensile-compressive cyclic strains. Finally, a simple impact apparatus is utilized to intentionally damage a plate. The intention of this setup is not to represent a specific health monitoring application but rather as a means of conveniently introducing permanent deformation and penetrations in a plate. The sensing skin will be used to image both the location and severity of the residual deformation and plate penetrations. This paper concludes with the sensing skin results generalized and a discussion of the possibility for using it in other health monitoring applications. 


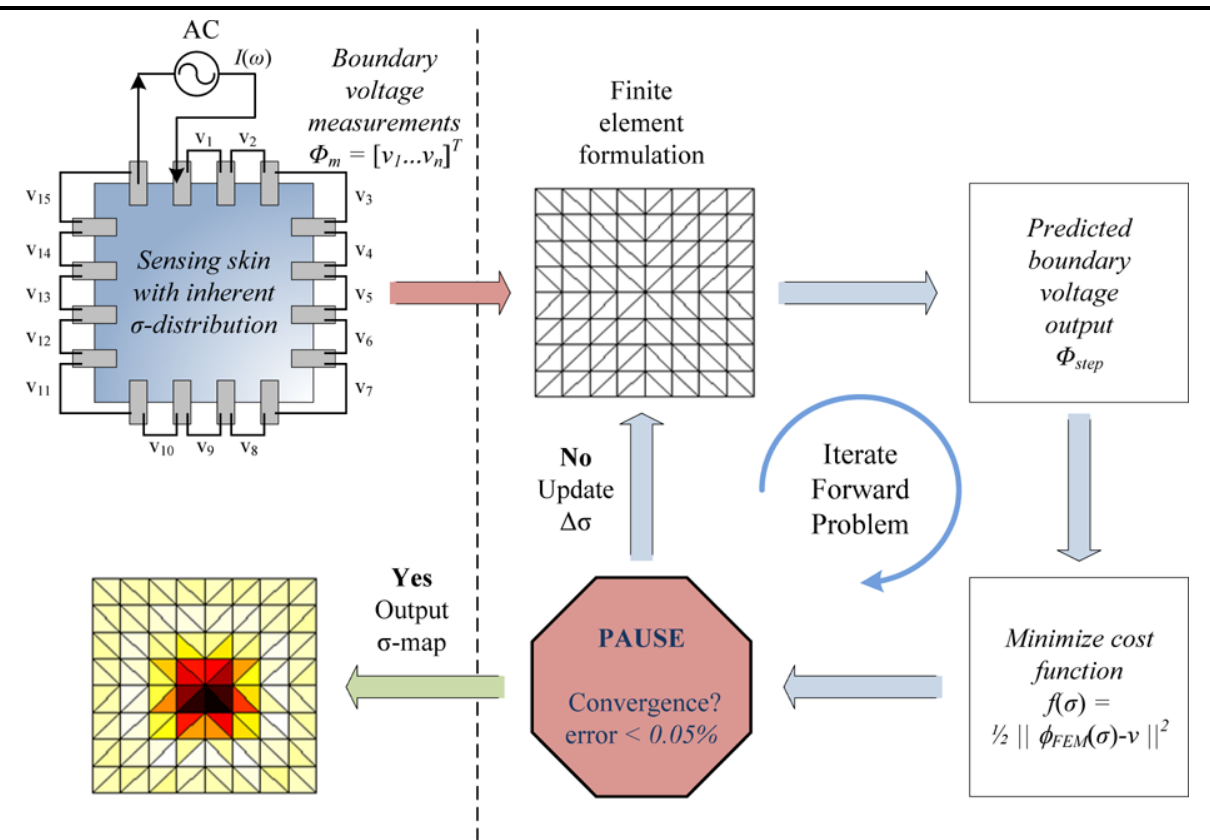

Fig. 1 A schematic illustrating the electrical impedance tomography spatial conductivity reconstruction process. The left side of the schematic shows the nature of the inverse problem (i.e., using boundary electrical measurements and known current inputs to find the conductivity distribution of the sensing skin). In order to solve for the

\section{Electrical Impedance Tomography}

In short, electrical impedance tomography reconstructs a material's two- or three-dimensional spatial conductivity distribution using boundary input-output currentvoltage measurements. A discrete set of equidistantlyspaced boundary electrodes are instrumented along the material of interest. Experimentally, an electrical current applied to a pair of boundary electrodes cause electric potential to develop across all the remaining electrodes. If the electrical conductivity of the body is known, the developed boundary electrical potential can also be predicted (the forward problem). However, EIT is an inverse problem that seeks to determine the spatial conductivity of an unknown body based on redundant input-output experimental boundary electrode measurements. Thus, upon obtaining a set of boundary electrode current-voltage measurements, an iterative reconstruction algorithm is employed to estimate the material's spatial conductivity distribution (the inverse problem). At the end of the iteration process, the forward problem is executed again to output the estimated spatial conductivity map of the sensing skin. An overview schematic of the EIT process is shown in Fig. 1. spatial conductivity distribution, an iterative process utilizing the finite element method is employed to repeatedly solve the forward problem until the solution converges (minimization of the cost function) as shown on the right side of the schematic

\subsection{Forward Problem: Background and Theoretical Foundations}

Given any two- (2D) or three-dimensional (3D) linear isotropic medium $(\boldsymbol{\Omega})$ described by a time-invariant conductivity distribution $(\sigma)$, Faraday's Law of Induction states,

$\nabla \times \boldsymbol{E}=-\frac{\partial}{\partial t} \boldsymbol{B}$

and Ampere's Law states,

$\nabla \times \boldsymbol{H}=\sigma \boldsymbol{E}+\boldsymbol{J}^{s}$

where $\boldsymbol{E}$ is the electric field, $\boldsymbol{H}$ is the magnetic field strength, $\boldsymbol{B}$ is the magnetic field $\left(\boldsymbol{B}=\mu_{0} \boldsymbol{H}\right), \mu_{0}$ is the magnetic permeability, and $\boldsymbol{J}^{S}$ is the current density of current sources within the body $\boldsymbol{\Omega}[21,22]$. By neglecting the magnetic field when using static or low-frequency currents, (1) and (2) can be combined to yield (3), which is termed the Laplace Equation. This equation is widely used to model the flow of electrical current and the development of electrical potential within conductors and semiconductors:

$\nabla \cdot(\sigma \nabla \phi)=I$

Here, it is assumed that current $I$ is neither supplied nor generated within $\boldsymbol{\Omega}$ and hence, the right hand side of the Laplace equation is set to zero. The flow of current within the body 
is therefore due to current supplied at the boundary of $\boldsymbol{\Omega}$. For a two-dimensional body (e.g., idealized thin film materials) with direct current (DC) applied to the body's boundary, (3) can be rewritten as the 2D Laplace equation (4) to describe current flow at every location within $\boldsymbol{\Omega}$ (based on a two-dimensional $(x, y)$ Cartesian coordinate system) [21].

$\nabla \cdot[\sigma(x, y) \nabla \phi(x, y)]=0$

Similarly, if an alternating current (AC) of cyclic frequency $\omega$ is applied to the medium, then the 2D Laplace equation is expressed as:

$\nabla \cdot[\gamma(x, y, \omega) \nabla \phi(x, y, \omega)]=0$

where the conductivity $(\sigma)$ of $\boldsymbol{\Omega}$ is replaced with the complex admittance, $\gamma$, to account for the body's conductivity, $\mathrm{AC}$ signal frequency, and material permittivity $(\varepsilon)$ as shown in (6).

$\gamma=\sigma+i \omega \varepsilon$

However, it is generally assumed that the applied electric signal's frequency is sufficiently low, such that $\omega \varepsilon$ is infinitesimally small and $\gamma \approx \sigma$. In that case, (4) is generally adopted in the EIT formulation. From a continuum model's perspective, it is assumed that there are no discrete electrodes and that the applied current is defined as a continuous function along the body boundary, $\partial \boldsymbol{\Omega}$ [21]. Regardless, if the potential of the boundary $(\phi)$ can be measured, the Dirichlet boundary condition states that

$\phi(x, y)=v(x, y) \quad$ at $\partial \boldsymbol{\Omega}$

where $v$ is the measured boundary voltage (or potential) drop. In addition, if the sum of input and output current, $I$, along the entire medium's boundary is zero (i.e., if the line integral of current along $\partial \boldsymbol{\Omega}$ is zero), then Neumann's boundary condition is satisfied as shown in (8).

$$
\oint I(\omega) \partial \boldsymbol{\Omega}=0
$$

Herein, the combination of the 2D Laplace equation (4) and Dirichlet-Neumann boundary conditions ((7) and (8)) is referred to as the forward problem for the EIT continuum model. The forward problem guarantees that the boundary potential $(v)$ can be determined theoretically via a known and continuous boundary current function applied to a body's boundary, $I_{\mid \partial \Omega}$, where the body is characterized by a conductivity distribution function, $\sigma(x, y)$ [23].

\subsection{Finite Element Method (FEM) Formulation}

Often, the EIT forward problem cannot be solved in the continuum domain since a continuum spatial conductivity distribution function and continuum applied boundary current function are practically unobtainable. As a result, a discretized weak form solution to the forward problem (Sect. 2.1) can be obtained using variational mechanics and techniques such as the finite element method (FEM). Here, FEM discrete elements of constant properties (e.g., conductivity) are employed to approximate the continuous 2D conductivity distribution function $(\sigma)$ [21, 24]. Similarly, as opposed to relying on a continuous boundary current function which is also practically unobtainable, the complete electrode model, which discretizes the boundary into a finite number of electrodes $(L)$, is implemented for finite element analyses [21, 25].

For the FEM formulation employed in this study, $n$ triangular elements with linear basis functions, $w_{i}$, are employed. The potential along the boundary of each element $\left(\left.\phi\right|_{\partial \boldsymbol{\Omega}_{\mathbf{E}}}\right)$ can then be obtained by taking the sum of the three nodal (i.e., the vertices of the triangular element) potentials of each $i$ th element multiplied by each of its corresponding basis functions as shown in (9).

$\left.\phi\right|_{\partial \boldsymbol{\Omega}_{\mathbf{E}}}=\sum_{i=1}^{3} \phi_{i} w_{i}$

Since the basis functions are not differentiable at the $i$ th node, the weak formulation of the Laplace equation is derived using variational methods:

$\int V[\nabla \cdot(\sigma \nabla \phi)] d V=0$

where $V$ is an arbitrary function and (10) is integrated over the body $\boldsymbol{\Omega}$. The combination of the weak form of the Laplace equation (10), the discretization of potential (9), and the Dirichlet-Neumann boundary conditions, results in a linear set of equations. In matrix format, the global set of linear equations is rewritten as:

$\left[\begin{array}{cc}\mathbf{A}_{M}+\mathbf{A}_{Z} & \mathbf{A}_{W} \\ \mathbf{A}_{W}^{\mathrm{T}} & \mathbf{A}_{D}\end{array}\right]\left[\begin{array}{c}\Phi \\ \boldsymbol{E}\end{array}\right]=\left[\begin{array}{l}0 \\ I\end{array}\right]$

where $\Phi=\left(\phi_{1}, \ldots, \phi_{n}\right)^{\mathrm{T}}$ is a vector that includes all elemental nodal potentials, $\boldsymbol{E}=\left(v_{1}, v_{2}, \ldots, v_{p}\right)^{\mathrm{T}}$ is a vector that contains all measured boundary electrode voltages, and $\mathbf{A}$ is a square matrix calculated from the linear basis functions $(w)$ and element conductivities $(\sigma)$ [19]. Thus, (11) provides the foundation for determining the measured boundary voltage, $\boldsymbol{E}$, due to the application of a known boundary current, $I$, to the numerically meshed body. The reader is referred to Hou, et al. [20] for a more detailed discussion regarding the implementation of triangular elements and the finite element method for electrical impedance tomography of thin film composites. 
2.3 Inverse Problem: Electrical Impedance Tomographic Conductivity Mapping

In short, electrical impedance tomography relies on a complete set of current injections and voltage measurements along a body's boundary (i.e., at its electrodes) to reconstruct its spatial conductivity distribution (herein termed the inverse problem and illustrated in Fig. 1). By its nature, the aforementioned inverse problem is ill-posed such that a unique solution may not exist for a given data set $[19,21]$. Therefore, current is injected on all possible sets of adjacent electrodes, with voltage measurements taken so as to create an over-determined data set. The inverse problem then seeks to determine the optimal spatial conductivity distribution by minimizing the difference between predicted boundary electrode potentials (as calculated by an assumed $\sigma$ distribution based on the FEM model) with actual experimental boundary voltage measurements, $\boldsymbol{E}$. More specifically, each step of the iterative inverse problem updates each FEM element's conductivity and solves the forward problem to determine the set of predicted boundary potentials. The predicted boundary potentials are compared to experimental boundary voltage measurements, and the EIT inverse problem seeks to minimize a quadratic least-squares objective function such as (12) [23].

$f(\sigma)=\frac{1}{2}\left\|\left.\phi(\sigma)\right|_{\partial \boldsymbol{\Omega}}-v\right\|^{2}$

Here, to solve the EIT spatial conductivity reconstruction, a standard regularized Gauss-Newton algorithm is employed to minimize a similar but augmented least-square functional that takes into account regularization. The regularized Gauss-Newton algorithm is selected for its computational efficiency and convergence properties; however, it should be mentioned that convergence is dependent on the assumptions made regarding the initial spatial conductivity of the 2D body [19]. The iterative algorithm is executed until the difference between the predicted boundary potential, $\phi(\sigma)$, and experimental boundary voltage measurements are within an acceptable error threshold (i.e., when $f(\sigma) \leq 0.05 \%)$. Upon convergence of the iterative Gauss-Newton inverse problem (i.e., when the error threshold criterion is met), the final conductivity map is derived. Unlike techniques such as backprojection which only provide relative spatial conductivity changes between successive EIT boundary electrical measurements, this algorithm reconstructs the absolute film conductivity and has been verified experimentally by Hou, et al. [20]. In fact, in the recent study conducted by Hou, et al. [20], EIT is employed to estimate the spatial conductivity maps of $25 \times 25 \mathrm{~mm}^{2}$ (SWNT-PSS/PVA $)_{n}$ nanocomposites. In that study, a thin film is cut into 16 square pieces to experimentally measure each square's surface conductivity. The maximum er- ror observed between EIT-estimated and experimental measurement is found to be $2.1 \%$. The small experimental error obtained suggests that most of the conductivity variations resulting from EIT spatial conductivity mapping can be attributed to contact impedance uncertainties and measurement error during surface probing [20].

\section{Experimental Details}

\subsection{Experimental Materials}

Single-walled carbon nanotubes (HiPCO) are purchased from Carbon Nanotechnologies, Inc. Polyelectrolyte species such as poly(sodium 4-styrene sulfonate) (PSS, $M_{w} \approx$ 1,000,000) and poly(vinyl alcohol) (PVA) are obtained from Sigma-Aldrich. To ensure repeatability between fabricated specimens, all solutions have been made with Milli-Q $18 \mathrm{M} \Omega$ deionized water. All remaining chemical reagents and supplies (e.g., glass microscope slides) are from FisherScientific. Metallic plate elements such as aluminum 6061T6 alloy are purchased from ALRO Metals Plus, Inc. (Ann Arbor, MI). Finally, colloidal silver paste and copper tape used for EIT boundary electrodes are from Ted Pella, Inc.

\subsection{Layer-by-Layer Sensing Skin Fabrication}

In this study, a layer-by-layer self-assembly technique is employed for fabrication of the proposed sensing skin. The LbL technique is simple, low cost, and versatile, capable of incorporating a wide variety of nanomaterials and polyelectrolyte species for the design of next-generation multifunctional nanocomposites [26]. Mechanically, nanocomposites can be tailored with properties such as high strength, stiffness, and ductility through the embedment of single-walled carbon nanotubes within a bulk polyelectrolyte matrix as have been demonstrated by Mamedov, et al. [15] and Loh [27].

In short, the LbL thin film fabrication process is based upon the sequential adsorption of oppositely charged polyelectrolyte and nanomaterial species to form homogeneous percolated nanostructures $[26,28]$. In this study, two oppositely-charged solutions are used: 1) negativelycharged SWNTs dispersed in 1.0 wt. \% poly(sodium 4styrene sulfonate) and 2) positively-charged 1.0 wt. \% poly(vinyl alcohol). Prior to LbL, non-covalent steric stabilization (i.e., stable solutions) of SWNTs is achieved by subjecting SWNTs in $1.0 \mathrm{wt}$. \% PSS $\left(0.80 \mathrm{mg} \mathrm{mL}^{-1}\right)$ to $180 \mathrm{~min}$ of ultrasonication bath treatment $(135 \mathrm{~W}, 42.0 \mathrm{kHz})$ followed by $90 \mathrm{~min}$ of high-energy tip-sonication $(3.178 \mathrm{~mm}$ tip, $150 \mathrm{~W}, 22.0 \mathrm{kHz})[16,17]$. While a variety of surfactants and polymers such as sodium dodecyl sulfate (SDS), sodium dodecylbenzene sulfonate (NaDDBS), PSS, among others, have been shown to provide adequate nanotube suspension, 


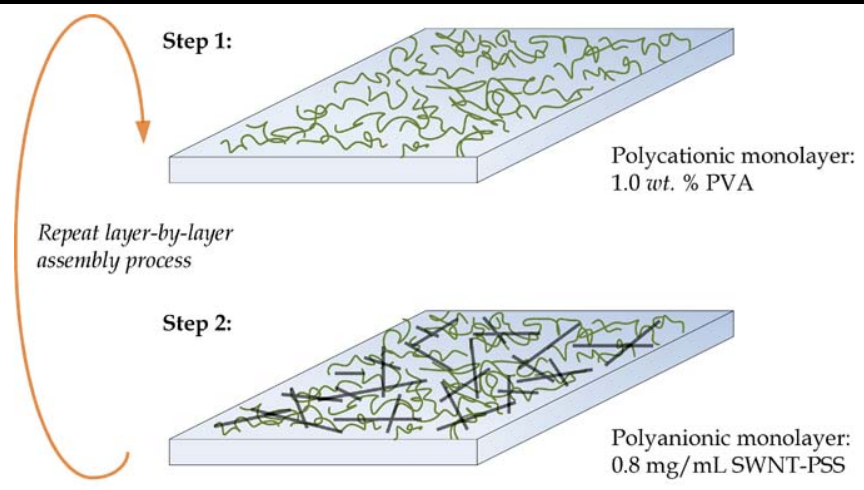

(a)

Fig. 2 (a) Schematic showing the sequential deposition of polycationic (PVA) and polyanionic (SWNT-PSS) monolayers during the layer-by-layer nanocomposite fabrication process. (b) A photograph

high molecular weight polyelectrolytes (i.e., PSS) not only facilitate dispersion [29] but also mechanically reinforce the final nanocomposite films.

Immediately prior to sensing skin fabrication, substrates (either glass or aluminum 6061-T6 alloy) surfaces are conditioned for optimal nanocomposite deposition and experimental testing. For instance, glass microscope slides are treated with piranha solution $\left(3: 7 \mathrm{H}_{2} \mathrm{SO}_{4}: \mathrm{H}_{2} \mathrm{O}_{2}\right)$ to clean and remove surface impurities such as oil, microbes, among others $[16,17]$. On the other hand, metallic plates are first cleaned with acetone to remove oil residue and then spraycoated with a thin uniform layer of primer to electrically isolate the sensing skins from the conductive substrates.

Upon completion of substrate preparation, the LbL process begins by immersing the substrate in the polycationic $1.0 \mathrm{wt}$ \% PVA solution for $5 \mathrm{~min}$. The substrate is then rinsed with $18 \mathrm{M} \Omega$ Milli-Q deionized water for $3 \mathrm{~min}$ and dried with compressed air for $10 \mathrm{~min}$. Then, the substrate, along with its adsorbed PVA monolayer, is dipped into the aforementioned SWNT-PSS suspension for $5 \mathrm{~min}$, followed by rinsing ( $3 \mathrm{~min})$ and drying $(10 \mathrm{~min})$. This process completes the adsorption of one nanocomposite bilayer and is repeated 50 or 100 times to achieve a mechanically-strong thin film sensing skin (Fig. 2). Herein, the sensing skins will be referred to as (SWNT-PSS/PVA) thin films, where SWNT-PSS and PVA represents the two oppositely-charged LbL constituents, while $n$ denotes the number of bilayers. For a more detailed discussion on layerby-layer self-assembly, the reader is referred to Decher and Schlenoff [26] or previous studies conducted by Loh, et al. $[16,17]$.

\subsection{EIT Electrode Preparation and Data Collection}

As opposed to two- or four-point surface conductivity measurements, electrical impedance tomography relies on a series of boundary electrical measurements for reconstructing

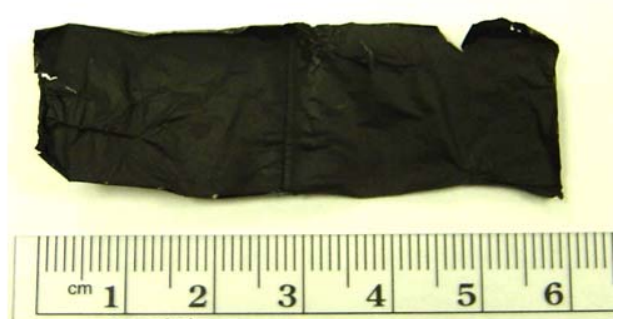

(b) of a free-standing (SWNT-PSS/PVA) 200 thin film etched from its glass substrate (using hydrofluoric acid)

the $2 \mathrm{D}$, or even $3 \mathrm{D}$, spatial conductivity image of any body, $\boldsymbol{\Omega}$ (as described in Sect. 2). Prior to data collection, sensing skins are instrumented with 16 or 32 electrodes around their boundaries (with equal number of electrodes equidistantly spaced along each boundary of the square/rectangular sensing skin specimens as shown in Figs. 3a, 4a, and 5a). For skins deposited on glass substrates for resolution analysis (Sect. 3.4) and strain sensing validation (Sect. 3.5), controlled electrode placement is achieved by using header pins with a fixed spacing. Colloidal silver paste is used to electrically connect the header pins to the nanocomposite surface. On the other hand, electrodes for impact damage identification (Sect. 3.6) are established using 32 copper tape electrodes; minimization of contact impedance is accomplished by drying colloidal silver paste between the copper tape and nanocomposite skin. The colloidal silver paste is allowed to dry for six hours prior to any experimental testing.

In order to obtain the reconstructed EIT spatial conductivity image, current flow is introduced into the skin at two adjacent electrodes on the skin boundary, while voltage is measured at all remaining electrodes. In this study, the neighboring or adjacent electrode measurement technique is employed [21]. Experimentally, a Keithley 6221 current generator is commanded to inject a regulated $\mathrm{AC}$ or DC current into a pair of adjacent electrodes, while a National Instruments (NI) data acquisition system (DAQ) is employed to measure the potential drop across all other adjacent electrode pairs. Using the neighboring electrode measurement technique, a total of $L(L-1) / 2$ measurements are derived (where $L$ is the number of electrodes). While this technique has been demonstrated to produce non-uniform current density within the body leading to reduced sensitivity to conductivity changes located away from the boundary [21], this technique is implemented for its simplicity and fast data acquisition properties. Furthermore, it will be demonstrated in Sect. 4 that the high strain sensitivity of the 


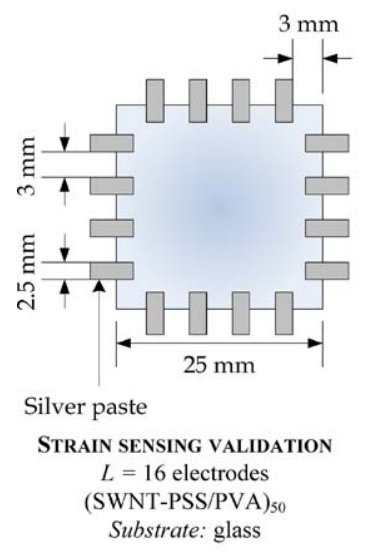

(a)

Fig. 3 (a) A schematic illustrating the EIT electrode configuration for strain sensing validation. (b) (SWNT-PSS/PVA) 50 thin films are deposited on $25 \times 25 \mathrm{~mm}^{2}$ glass substrates. The sensing skin is epoxy-

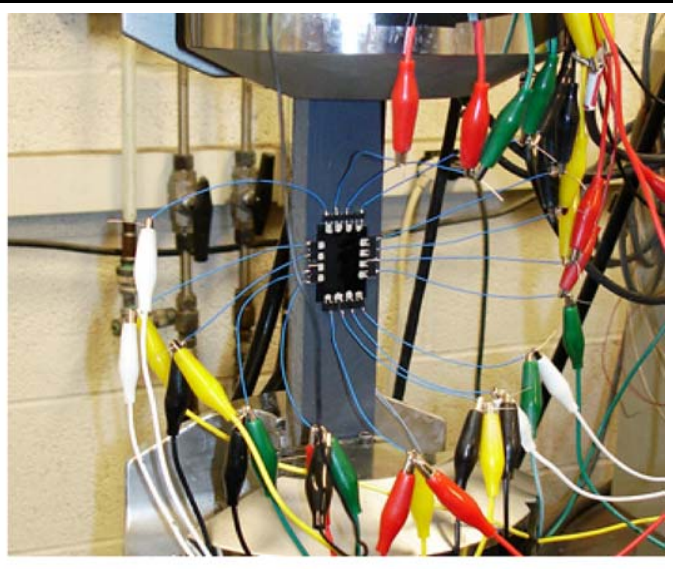

(b)

mounted onto PVC Type I coupons and mechanically loaded in an MTS-810 load frame. The load frame is displacement-controlled to mechanically load the thin film to $\pm 2,000 \mu \varepsilon$ or $\pm 5,000 \mu \varepsilon$

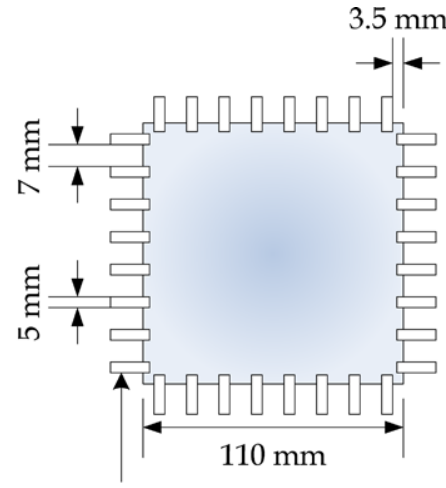

Copper tape \& Silver paste

IMPACT DAMAGE DETECTION

$L=32$ electrodes

(SWNT-PSS/PVA) $)_{50}$

Substrate: aluminum

(a)

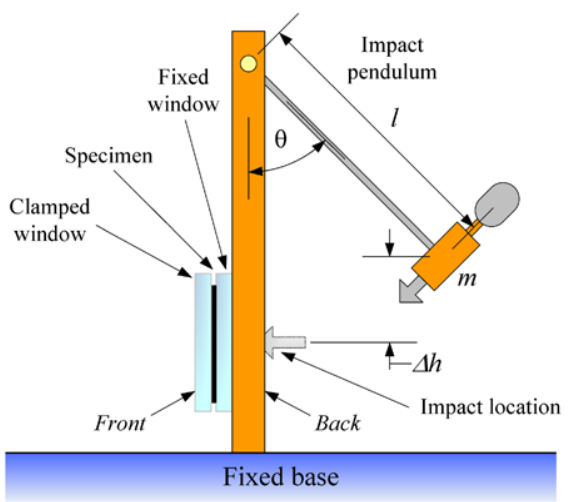

(b)

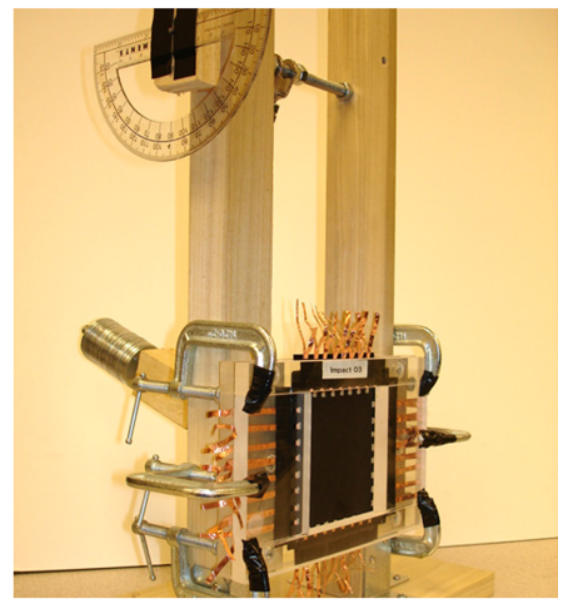

(c)
Fig. 4 (a) A schematic illustrating the EIT electrode configuration for impact damage detection. (b) The impact testing apparatus is equipped with an adjustable arm pendulum (of length, $l$ ) and mass block $(m)$. By controlling the height $(\Delta h)$ of the pendulum arm, a specific amount of potential energy can be transferred to the plate during impact.

proposed nanocomposite skins (i.e., relative to traditional sensors, such as metal-foil strain gages), are unaffected by the neighboring electrode measurement technique.

\subsection{Sensing Skin Resolution Analysis}

It has been shown in (4) that the two-dimensional Laplace equation is capable of modeling the flow of electrical current and the development of a boundary potential via a continuum conductivity distribution function $(\sigma)$. With a (c) (SWNT-PSS/PVA) $)_{50}$ skins deposited on aluminum 6061-T6 alloy are mounted onto an impact-loading test apparatus. Fixed boundary conditions are ensured by clamping a plexiglass frame over the skin and plate

continuum approach to electrical impedance tomography, the sensing skin's sensitivity and resolution to damage features are governed by inherent nanocomposite sensor properties. However, for practical applications, the EIT conductivity mapping approach employed herein relies on a discretized weak form solution using the finite element method (Sect. 2). Solution to the discretized EIT inverse problem yields a computed conductivity map comprised of a set of finite elements, where each element is described by an average conductivity representation of real space. In particular, 


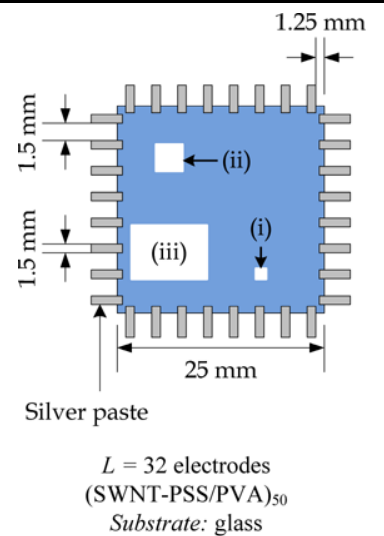

(a)

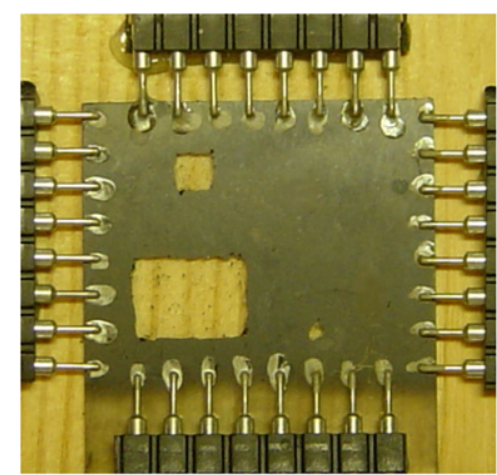

(b)

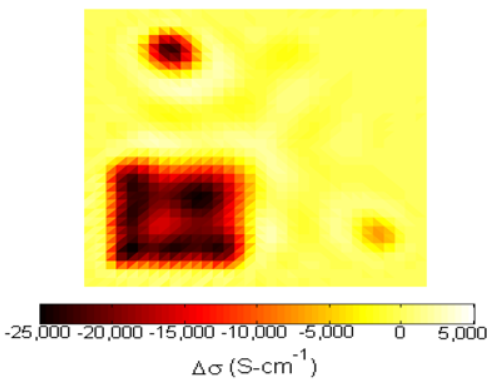

(c)
Fig. 5 (a) A schematic showing a (SWNT-PSS/PVA) 50 nanocomposite fabricated onto a glass substrate with three mechanically-etched windows. Eight EIT electrodes (header pins and colloidal silver paste) are established on each of the four boundaries of the $25 \times 25 \mathrm{~mm}^{2}$ thin film. (b) A corresponding photograph of an etched (SWNTPSS/PVA) 50 specimen. (c) EIT spatial conductivity map showing the change in conductivity due to etching

$25 \mathrm{~mm}^{2}$ glass substrates are affixed onto PVC (polyvinyl chloride) Type I tensile coupons (31 cm long, $4 \mathrm{~cm}$ wide, $2 \mathrm{~cm}$ thick) via $\mathrm{CN}-\mathrm{Y}$ post-yield epoxy (Tokyo Sokki Kenkyujo). Upon sufficient drying of the epoxy (6 hours), eight header pins are also epoxy-mounted to each of the four sides of the square skin (Fig. 3a). Colloidal silver paste is dried between adjacent pairs of header pins to form equidistantly-spaced EIT boundary electrodes. Finally, each (SWNT-PSS/PVA) 50 sensing skin (on glass), along with the PVC coupon, is mounted in an MTS-810 load frame where it is programmed to execute a tensile-compressive cyclic load pattern to $\pm 2,000$ or $\pm 5,000 \mu \varepsilon$ at a fixed load rate of $50 \mu \varepsilon \mathrm{s}^{-1}$ so as to prevent cracking of the glass substrate (Fig. 3b). The load frame is paused at $1,000 \mu \varepsilon$ or $2,500 \mu \varepsilon$ intervals while the EIT DAQ is commanded to inject current and measure boundary electrode potentials as described in Sect. 3.3.

\subsection{Distributed Impact Damage Detection}

As mentioned in Sect. 3.3, (SWNT-PSS/PVA) 50 deposited on $110 \times 110 \mathrm{~mm}^{2}$ primer-coated aluminum 6061 -T6 alloys $(0.75 \mathrm{~mm}$ thick) are used for impact-based damage detection validation tests (Fig. 4a). Clearly, impact damage is common in structures such as aerospace systems (e.g., impact damage is common on the space shuttle during launch) and naval structures (e.g., blast-induced hull penetrations). However, in this study, impact testing is only used to control the introduction of permanent strain in a structural specimen. In addition, large impacts can be used to penetrate the skin and structure. The ability to clearly capture both permanent residual strain and structural penetrations by EIT-based sensing skins would suggest that the technology is applicable to many other damage scenarios such as for capturing fatigue cracking. 
In order to induce different thresholds of impact damage on the large substrate, a pendulum impact testing apparatus is constructed in the laboratory. The objective of this study is to employ a (SWNT-PSS/PVA)50 sensing skin to detect various magnitudes of controlled damage at distributed locations on an aluminum plate. The pendulum test structure consists of an adjustable swinging arm (variable between 20 to $30 \mathrm{~cm}$ ), a sharp aluminum tip (termed the striker), and a rod connected to the striker to hang washers for controlling the pendulum mass as shown in Fig. $4 \mathrm{~b}$ and $4 \mathrm{c}$. In addition, the test apparatus is configured such that the pendulum arm can be rotated between $0^{\circ}$ and $180^{\circ}$ to control the magnitude of the initial potential energy that is converted to the final impact-deformation strain energy (where $0^{\circ}$ corresponds to the situation when the tip of the striker is in contact with the skin specimen).

Following the layer-by-layer self-assembly methodology described in Sect. 3.2, sensing skins are directly deposited on both sides of the metallic plate specimens. The "back" side of the plate is where the impact striker physically contacts and impacts the skin, and the "front" side is the opposite non-contact face. Each skin-coated aluminum alloy specimen is mounted with fixed-fixed boundary conditions on the testing apparatus. Two sturdy plexiglass windows are securely mounted to the pendulum structure, and a total of six C-clamps are used to secure plate specimens between the two windows. The two clamped windows and C-clamps ensure that the skin-coated aluminum alloy specimens do not slip or displace during impact testing; thus, it can be assumed that the impact energy is transmitted entirely to the plate in the form of permanent deformation and strain energy. In this series of tests, the pendulum is adjusted to impact two plate specimens at various locations with different initial potential energies (i.e., the striker mounted on the pendulum is released at different initial angles and arm lengths). In order to demonstrate the sensing skins' ability to capture the location of damage and severity of impact (i.e., the amount of strain energy absorbed for plate deformation) across large structural surfaces, four impacts are induced per specimen. Finally, EIT conductivity mapping is performed to identify damage.

\section{Experimental Results and Discussion}

\subsection{Sensing Skin Resolution Analysis}

As detailed in Sect. 3.4, a (SWNT-PSS/PVA)50 nanocomposite is deposited onto a glass substrate and mounted with 32 boundary electrodes (Fig. 5a). As can be seen in Fig. 5b, the electrode placement was not precise with the top- and bottom-most electrodes on the left and right sides nearly inline with the top and bottom side electrodes. Nonetheless,
EIT analysis can still be applied assuming equidistant electrode spacing consistent with Fig. 5a. First, an initial EIT spatial conductivity map is obtained to serve as the baseline. Upon mechanical etching to remove the thin film at three distinct rectangular regions, EIT is executed again to compute the relative spatial conductivity change (Fig. 5c). From Fig. 5c, it can be clearly observed that the sensing skin successfully identifies three distinct rectangular regions (i.e., (i), (ii), and (iii) corresponding to Fig. 5a) with a significant drop in conductivity due to the removal of the nanocomposite skin. While the sensing skin is capable of accurately identifying the size and location of etched regions (ii) and (iii), it can be seen from Fig. 5c that the magnitude of conductivity change near etched window (i) is smaller than regions (ii) and (iii). Since each EIT boundary electrode and electrode spacing are both $1.5 \mathrm{~mm}$ wide, the size of this damage feature (i.e., region (i) is $1 \times 1 \mathrm{~mm}^{2}$ ) is smaller than a pair of triangular finite elements within the reconstructed EIT spatial conductivity map. As a result, damage feature (i) represents the detection limit or resolution of the sensing skin. It is anticipated that the sensing skin is relatively insensitive to damage features smaller than the size of one finite element of the reconstructed EIT image.

Despite being able to identify the size and geometry of etched film regions, determination of etched location is extremely sensitive to boundary electrode placement (i.e., alignment of electrodes equidistantly spaced along each side of the thin film). Upon comparing the specimen photograph and EIT spatial conductivity map in Fig. 5, it can be observed that the predicated damage location is not exact. In fact, the smallest square damage feature in Fig. $5 \mathrm{~b}$ is rightshifted in the EIT spatial conductivity map of Fig. 5c. This misalignment is obtained due to the slight inaccurate placement of header pins along the thin film (Fig. 5b), while the finite element formulation to solve the EIT inverse problem assumes perfect electrode placement. Nevertheless, upon comparing the size and geometry of predicted spatial conductivity changes to each of the etched regions one at a time, good correlation is observed.

In order to provide a more quantitative analysis of the sensing skin's accuracy in identifying conductivity changes at each area where the nanocomposite skin has been etched, the photograph (Fig. 5b) and EIT spatial conductivity map (Fig. 5c) of the skin is assembled into normalized $n$-by- $m$ matrices $[\mathbf{P}]$ and $[\mathbf{C}]$, respectively. It should be noted that this analysis is only conducted in the vicinity of each region and not for the entirety of the full film surface. In this case, $[\mathbf{P}]$ is a binary representation of the actual specimen photo; $P_{x y}$ is 1 at locations where the nanocomposite is intact, and $P_{x y}$ is 0 where the thin film is mechanically etched. On the other hand, each element of the conductivity map matrix [C] is scaled between 0 and 1 to represent the computed sensing skin conductivity (where zero corresponds to the nonconductive case, and unity represents maximum electrical 


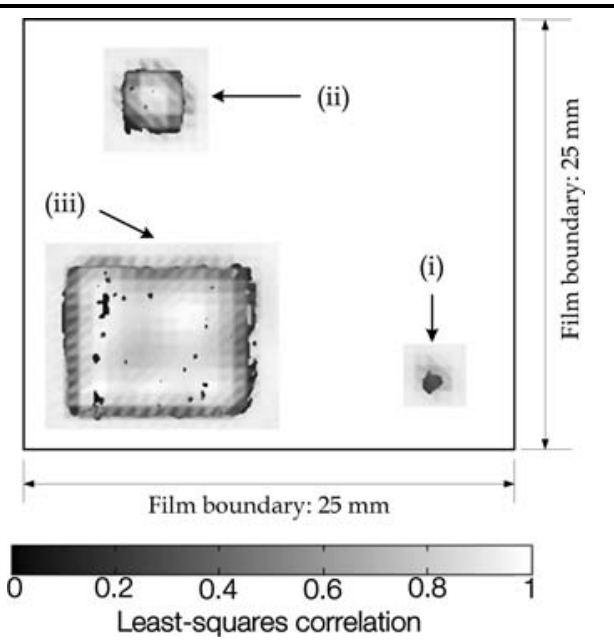

Fig. 6 Least-squares correlation matrix $[\mathbf{E}]$ is computed at the three rectangular areas where the skin has been etched. The plot shows the differences between the computed EIT spatial conductivity map (Fig. 5c) and the actual specimen (Fig. 5b). It should be noted that perfect correlation is shown as white $\left(E_{x y}=1\right)$

conductivity). Thus, the correlation between the computed EIT conductivity map and specimen is obtained by computing the least-squares error matrix $[\mathbf{E}]$ between each pair of corresponding pixels as shown in (14):

$$
\begin{aligned}
& {\left[\begin{array}{ccc}
E_{11} & \cdots & E_{1 m} \\
\vdots & \ddots & \vdots \\
E_{n 1} & \cdots & E_{n m}
\end{array}\right]} \\
& \quad=\left[\begin{array}{ccc}
1-\sqrt{\left(P_{11}-C_{11}\right)^{2}} & \cdots & 1-\sqrt{\left(P_{1 m}-C_{1 m}\right)^{2}} \\
\vdots & \ddots & \vdots \\
1-\sqrt{\left(P_{n 1}-C_{n 1}\right)^{2}} & \cdots & 1-\sqrt{\left(P_{n m}-C_{n m}\right)^{2}}
\end{array}\right]
\end{aligned}
$$

By definition, each element of $[\mathbf{E}]$ also spans between 0 and 1, such that maximum correlation is obtained when $E_{x y}$ is 1 (and $E_{x y}$ is 0 when there is no correlation).

The least-squares error matrix at each area where the sensing skin has been etched is computed and plotted together in Fig. 6 to illustrate the degree of correlation between the EIT conductivity map and specimen. It should be mentioned that each damage feature is compared independently, and the results from the correlation analyses are combined to produce Fig. 6. From Fig. 6, it can be seen that the sensing skin is capable of accurately identifying the size and geometry of different etched regions. Evaluation of correlation is achieved by computing the average least-squares error of each etched region's error matrix one at a time, $[\mathbf{E}]_{i}$, where $E_{\text {avg,(i) }}=0.69, E_{\text {avg,(ii) }}=0.76$, and $E_{\text {avg,(iii) }}=0.82$ (corresponding to regions (i), (ii), and (iii) respectively). The computed average correlation suggests that, as the etched feature size decreases from region (iii) to region (i), the degree of correlation also decreases (as also evident in Fig. 6).
At the limit where etched feature (i) is only $1 \times 1 \mathrm{~mm}^{2}$, it can be seen from Fig. 6 that the correlation obtained is only average. The lack of correlation obtained at etched region (i) is expected since each EIT boundary electrode is $1.5 \mathrm{~mm}$ wide and is larger than this particular damage feature. Thus, results from this analysis show that the sensing skin resolution is limited by EIT boundary electrode dimensions. Nevertheless, for spatial conductivity changes larger than each EIT boundary electrode, the sensing skin can accurately identify damage.

\subsection{Time-Invariant EIT Conductivity Mapping}

Previous studies conducted by Loh, et al. [16, 17] have validated (SWNT-PSS/PVA) ${ }_{n}$ films' change in electrical properties to applied strain. In general, nanocomposite electrical properties respond linearly to applied strain, where film resistivity $(\rho=1 / \sigma)$ is proportional to strain and has been modeled accurately using a parallel resistor-capacitor (RC) circuit [17]. The typical strain sensitivity $\left(S_{S}\right)$ is estimated to be approximately 2.0, comparable to those of traditional metal-foil strain gages and is calculated using (15) (in terms of conductivity) [16, 17],

$S_{S}=\left(\Delta \sigma / \sigma_{0}\right) / \varepsilon$

where $\Delta \sigma$ is the conductivity change due to applied strain $\varepsilon$, and $\sigma_{0}$ is the nominal (i.e., no strain) conductivity of the sensing film. It should be noted that the skin's strain sensitivity is expressed as a scalar parameter since these carbon nanotube-based nanocomposites are not biased towards certain loading directions. Instead, carbon nanotubes are deposited to form a randomly-percolated homogeneous thin film that changes its electrical conductivity in response to any applied in-plane strains with the resulting conductivity change is the combined magnitude of the localized strain tensor. In addition, the random network of percolated SWNTs permits current flow in all directions. Nevertheless, preferential unidirectional strain sensing is possible via alignment of nanotubes within the bulk polymeric matrix.

However, it has been determined that these LbL nanocomposites exhibit an undesirable exponential decrease in nominal film resistivity $\left(R_{0}\right)$ over time $(\sim 10 \mathrm{hrs})$ [16]. Nevertheless, the application of electrical impedance tomographic spatial conductivity imaging avoids having to account for the nanocomposite's time-variant nominal conductivity (or its inverse, resistivity). Results obtained from a study conducted by Loh, et al. [16] have demonstrated that the resistivity decay rate is proportional to applied current (for measuring film conductivity or resistivity), where a larger current input increases the decay rate. In addition, upon removal of the applied current source, the nominal conductivity of the film recovers to its initial pristine state. Thus, applied AC or DC current waveforms injected 
into two adjacent electrodes for EIT spatial conductivity mapping are limited in amplitude $(\sim 0.1 \mathrm{~mA})$ and duration $(\sim 1 \mathrm{~ms})$. In fact, experimental results have validated the time-invariant conductivity maps generated by repeated EIT reconstruction [20].

\subsection{Linear Strain Sensing Validation}

To demonstrate that EIT-reconstructed spatial conductivity images also exhibit linear changes in average conductivity with applied strain $[16,17]$, sensing skin specimens are epoxy mounted to PVC Type I bars and mechanically loaded in a one-cycle tensile-compressive load pattern to $\pm 2,000$ and $\pm 5,000 \mu \varepsilon$ (Sect. 3.5). Figure 7 plots the set of sequential EIT spatial conductivity maps of an (SWNTPSS/PVA) 50 thin film strained at $1,000 \mu \varepsilon$ intervals during the application of a one-cycle tensile-compressive load pattern to $\pm 2,000 \mu \varepsilon$. From Fig. 7, it can be clearly identified that the overall change in skin conductivity varies inversely with applied strain (i.e., the negative change in conductivity changes in tandem with increasingly applied strain). In addition, the spatial conductivity maps obtained at every $1,000 \mu \varepsilon$ interval suggests uniform deformation and conductivity change with applied strain.

Although the overall spatial conductivity of the carbon nanotube-based composite changes uniformly with applied strain, non-uniform conductivity changes are observed at the right edge of the nanocomposite. In fact, a number of factors can cause the observed non-uniform conductivity changes.
For instance, localized lack of strain transfer can occur at locations where the sensing skin's substrate is poorly bonded to the PVC testing coupon. The lack of strain transfer would induce non-uniform strain in the sensing skin. It is also possible that the sensing skin is accurately monitoring the PVC testing coupon's non-uniform strain distribution due to misalignment of the testing coupon, load frame grip slippage, among others. A third factor could be thin film inhomogeneity that could lead to an anisotropic response of the thin film to applied strain. Finally, the observed non-uniformity may simply be a result of a numerical artifact in the EIT inverse solution. Further study is needed to determine the precise cause of the minor variation in the right side of the EITderived conductivity maps.

Nevertheless, to evaluate the skin's strain sensing performance (i.e., linearity and sensitivity), the average change in conductivity corresponding to all elements of the EIT conductivity map is computed and compared to the applied load pattern as shown in Fig. 8a. It can be seen from Fig. 8a that the negative change in average EIT skin conductivities are directly proportional to applied strain. In addition, the results obtained validate the sensing skin's ability to capture both tensile and compressive strains. By plotting the negative change in conductivity as a function of applied strain, it can be seen from Fig. $8 \mathrm{~b}$ that the proposed sensing skins exhibit linear strain sensing response. Furthermore, the slope of the least-squares fitted line for all specimens tested is computed to quantify the strain sensor sensitivity (15). The computed strain sensitivities $\left(S_{S}\right)$ presented in Fig. $8 \mathrm{~b}$ range from 3.2 to 6.5 , thereby suggesting higher sensitivity than

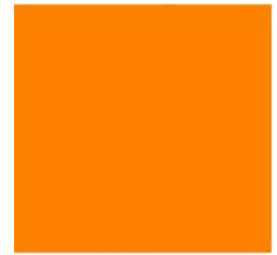

(a) $0 \mu \varepsilon$

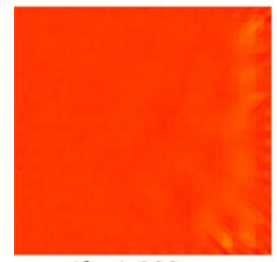

(f) $-1,000 \mu \varepsilon$

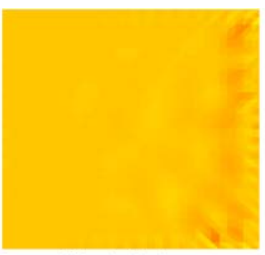

(b) $1,000 \mu \varepsilon$

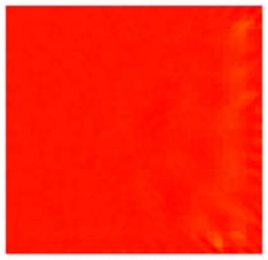

(g) $-2,000 \mu \varepsilon$

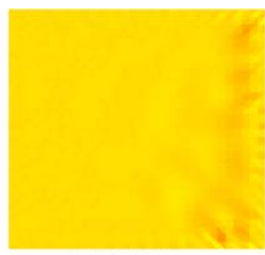

(c) $2,000 \mu \varepsilon$

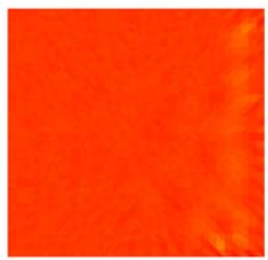

(h) $-1,000 \mu \varepsilon$

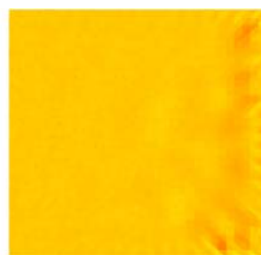

(d) $1,000 \mu \varepsilon$

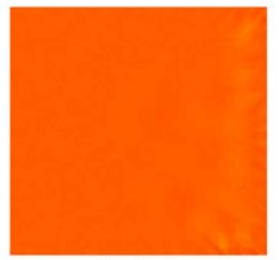

(i) $0 \mu \varepsilon$

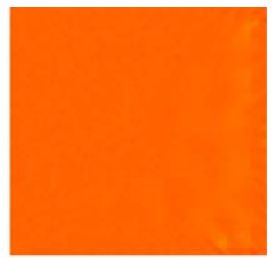

(e) $0 \mu \varepsilon$

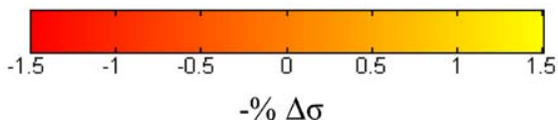

Fig. 7 (SWNT-PSS/PVA) 50 skins are deposited onto glass substrates and epoxy-mounted to PVC coupons for load testing. A one-cycle tensile-compressive load pattern $( \pm 2,000 \mu \varepsilon)$ is executed on specimen Skin 1. In (a) to (i), EIT spatial conductivity mapping is conducted at $1,000 \mu \varepsilon$ increments to monitor the sensing skin's $2 \mathrm{D}$ conductivity change. It can be seen that the sensing skin's negative percent change in conductivity (i.e., percent change in resistivity) varies in tandem with increasingly applied strain 

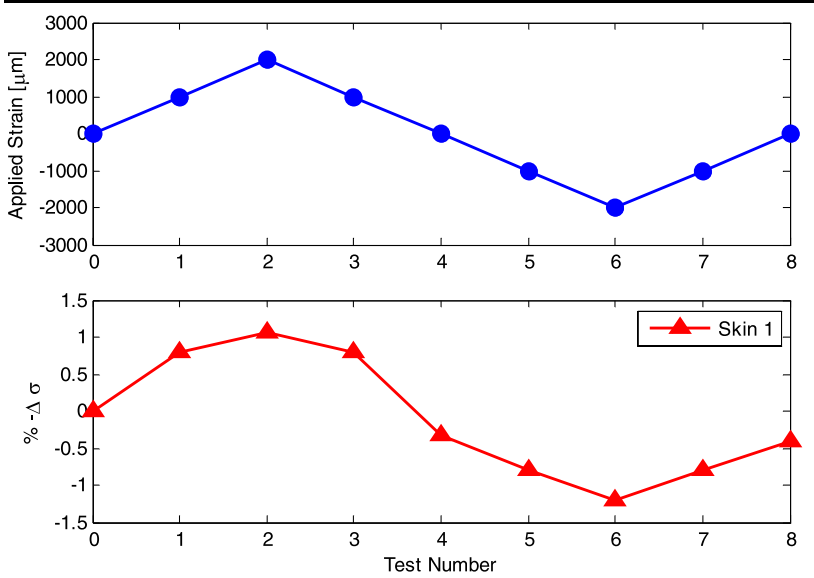

(a)

Fig. 8 (a) (Top) A one-cycle tensile-compressive load pattern to $\pm 2,000 \mu \varepsilon$ is applied to each sensing skin specimen. The load frame is paused at $1,000 \mu \varepsilon$ to allow EIT data acquisition. (Bottom) It can be seen that the strained sensing skin's percent change in conductivity changes in tandem with the applied strain. (b) Upon plotting the normalized negative change in average film conductivity $(\%-\Delta \sigma)$ against

traditional metal-foil strain gages $\left(S_{S} \approx 2\right)$. Thus, the sensing skin's high strain sensitivity and high resolution are ideally suited for structural monitoring applications to detect small changes in a structure's induced strains.

It should be noted that the variation in strain sensitivities is obtained from skins fabricated from different carbon nanotube concentrations and film thicknesses. Here, Skin 1 is fabricated from $0.5 \mathrm{mg} \mathrm{mL}^{-1}$ SWNT-PSS solution and is 100 bilayers; Skin 2 is fabricated from $0.5 \mathrm{mg} \mathrm{mL}^{-1}$ SWNTPSS solution and is 50 bilayers; and Skin 3 is fabricated from $0.8 \mathrm{mg} \mathrm{mL}^{-1}$ and is 50 bilayers. In an earlier study conducted by Loh, et al. [17], it has been determined that higher nanotube weight content within the nanocomposite and thinner films yield higher strain sensitivities.

\subsection{Spatial Impact Damage Detection}

This study is extended to detect spatially-distributed damage (i.e., permanent deformation and cracking) of metallic plate elements using the proposed LbL carbon nanotube-based skins. Unlike Sect. 4.3, sensing skins are directly deposited onto aluminum alloy plates; as a result, the strong adsorption and electrostatic forces between the skin and metallic substrate ensure complete strain transfer from impact. Using the experimental setup described in Sect. 3.6, four different magnitudes of impact are applied at various locations on each of the two plate specimens (herein referred to as IM-01 and IM-02). From Tables 1 and 2, it can be seen that the lowest applied impact energy is labeled as [i], while the highest impact energy is referred to as [iv]. Here, low levels of impact damage (i.e., damage states [i] and [ii]) correspond to

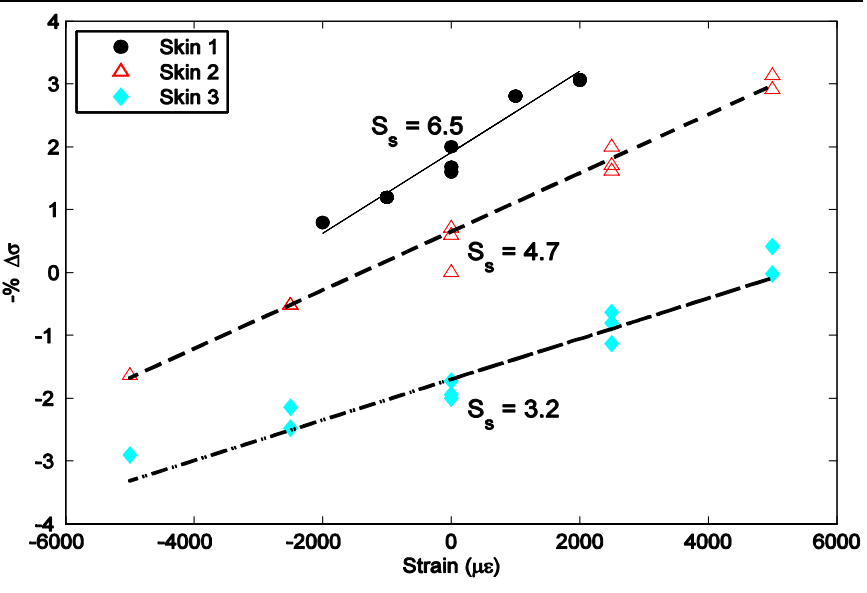

(b)

the applied strain for all skin specimens, the results confirm the skin's linear strain sensing performance. In addition, by fitting a least-squared line to the obtained data, the strain sensing sensitivity $\left(S_{S}\right)$ is approximately between 3.2 and 6.5. Note: The plots for Skin 1 and Skin 3 are translated in the $\pm y$-direction (by $2.5 \%$ ) for better visualization

permanent dents on the aluminum structure. This low level of damage is worthy of consideration since these defect sites serve as regions of stress concentration and origins of further crack propagation during repeated loading (e.g., in aircrafts, naval vessels, among others). Similarly, high levels of damage (i.e., damage states [iii] and [iv]) refer to severe damage states where the aluminum substrate is penetrated due to impact.

Prior to any induced impact damage, boundary electrical measurements are obtained and the EIT inverse problem is solved to reconstruct the baseline undamaged conductivity distribution of the pristine plate. Then, in order to evaluate the sensing skin's ability to detect both the location and severity of structural impact damage, the first plate specimen (IM-01) is mounted in the pendulum test apparatus (Fig. 4b) and impacted four times (as shown in Figs. 9a (front) and 9d (back)). Each of the four controlled impacts is conducted at different locations and have different initial impact energies (i.e., controlled by the arm length and initial pendulum angle) as listed in Table 1. Once all four impact damages have occurred, EIT spatial conductivity mapping is executed to obtain a "damaged" sensing skin conductivity map. From Figs. 9b (front) and 9e (back), it can be seen that the relative negative conductivity change for both the front and back sides clearly identify damage location and severity. From Sect. 4.3, it has been validated that higher levels of applied strain causes greater change in skin conductivity. With higher initial applied impact energies (e.g., damage locations [iii] and [iv]), the plate and skins undergo higher levels of deformation and strain. Thus, with greater initial impact energies (i.e., severe damage), the change in 
Table 1 Controlled impact

damage on skin- and

primer-coated aluminum plates for specimen IM-01
Table 2 Controlled impact damage on skin- and primer-coated aluminum plates for specimen IM-02

\begin{tabular}{lllll}
\hline Impact number & Location (front) & Arm length $(\mathrm{cm})$ & Pendulum angle $\left(^{\circ}\right)$ & Impact energy $(\mathrm{J})$ \\
\hline Impact-[i] & Top right & 24 & 20 & 0.09 \\
Impact-[ii] & Bottom left & 27.5 & 40 & 0.38 \\
Impact-[iii] & Bottom right & 27.5 & 60 & 0.81 \\
Impact-[iv] & Top left & 24 & 80 & 1.17 \\
\hline
\end{tabular}

\begin{tabular}{lllll}
\hline Impact number & Location (front) & Arm length $(\mathrm{cm})$ & Pendulum angle $\left({ }^{\circ}\right)$ & Impact energy $(\mathrm{J})$ \\
\hline First test (0 days) & & & & \\
Impact-[ii] & Middle right & 25.7 & 60 & 0.71 \\
Impact-[iii] & Bottom center & 28.5 & 90 & 1.63 \\
\multicolumn{2}{l}{ Second test (14 days) } & & & \\
Impact-[i] & Top left & 23.6 & 30 & 0.19 \\
Impact-[iv] & Center & 25.4 & 180 & 3.25 \\
\hline
\end{tabular}

\section{Front:}

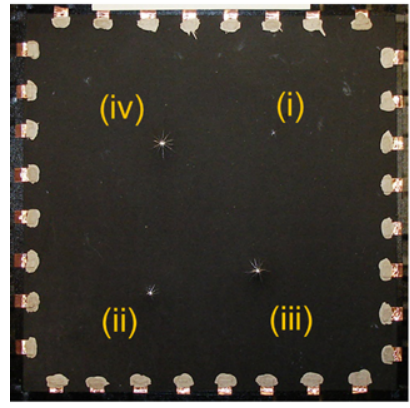

(a)

BACK:

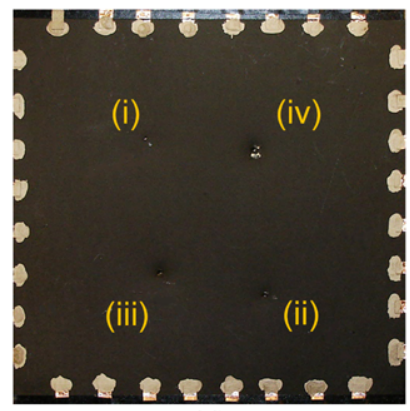

(d)

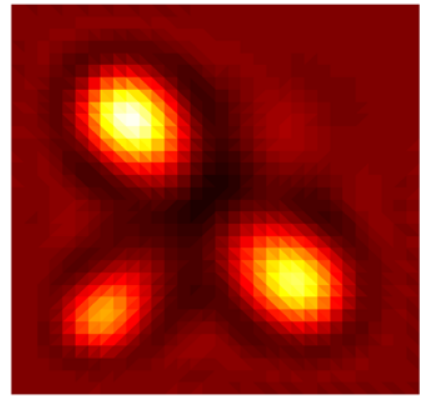

(b)

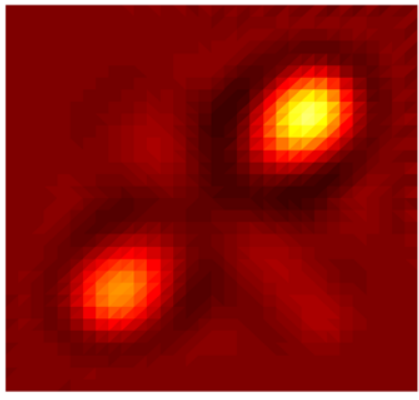

(e)

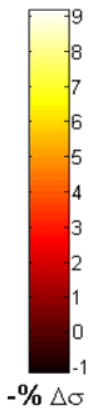

$-\% \Delta \sigma$

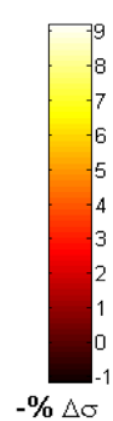

$-\% \Delta \sigma$

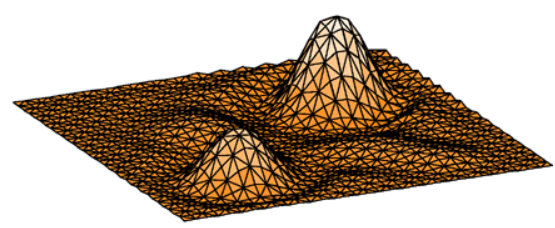

(f)
Fig. 9 Specimen IM-01: EIT is employed to reconstruct the spatial conductivity distribution of the skins after four different magnitudes of controlled pendulum impacts [(i)-(iv)] have occurred. Damage can be visually identified from photographs taken from the (a) front and (d) back sides of the plate. In addition, the reconstructed front-side (b) 2D and (c) 3D representation and back-side (e) 2D and (f) 3D representation EIT spatial conductivity images all verify the sensing skin's impact damage detection capabilities sensing skin conductivity is expected to be higher as shown in Figs. 9b (front) and 9e (back).

It should be noted that small damage thresholds (impact locations [i] and [ii]) are hard to detect in the 2D reconstructed EIT conductivity maps; however, damage is accu- rately detected but is masked within the color bar to better illustrate larger induced damages (impact locations [iii] and [iv]). To better make this point, the 2D maps are plotted in 3D to facilitate damage identification (see Figs. 9c and 9f). Upon computing the average change in skin conduc- 
tivity within the vicinity of induced impact damage for the front- and back-side skins, it can be observed from Fig. 10 that small levels of damage (i.e., dents on the aluminum

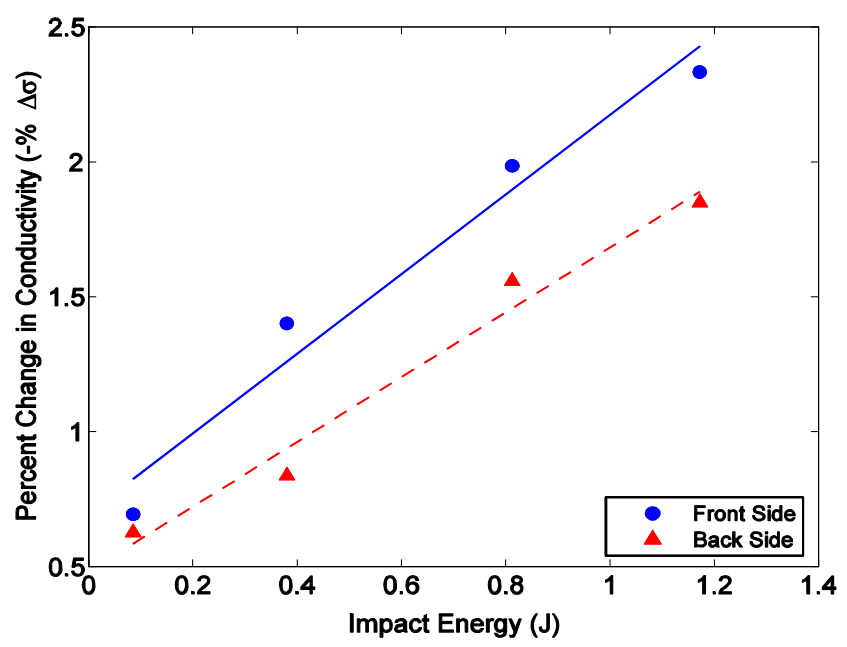

Fig. 10 The percent change in average film conductivity of specimen IM-01 increases near-linearly with increasing impact energy (as controlled by the initial potential energy of the impact test apparatus pendulum). In addition, the similarity in the fitted lines' slopes suggests that the sensing skins exhibits similar sensitivity to strain and impact plate comparable to the size of boundary electrodes) can be detected. In addition, the change in conductivity varies nearlinearly with respect to initial impact energy.

For sensing skins to demonstrate potential applicability for long-term structural health monitoring, it is important to begin characterization of sensor long-term performance. For instance, structural damage typically occurs continuously throughout the service life of engineered systems. Thus, in addition to being able to detect pristine and damaged structural states, it is worthwhile to investigate the sensing skin's response to damage induced over time. Thus, upon obtaining boundary electrical measurements for specimen IM-02 to determine the initial baseline conductivity map, two additional impact tests are conducted. First, two different magnitudes of pendulum hits (damage levels [ii] and [iii] tabulated in Table 2 and shown in Figs. 11a and 11d) are conducted at different locations on the aluminum alloy plate. Similarly, impact damage location and severity can be identified precisely from the 2D (Figs. 11b and 11e) and 3D (Figs. 11c and 11f) EIT conductivity images for the front and back sides of the plate, respectively.

Upon completion of the aforementioned test, the plate specimen is stored in ambient conditions for approximately 14 days prior to the next damage occurrence. Here, two ad-

\section{FronT:}

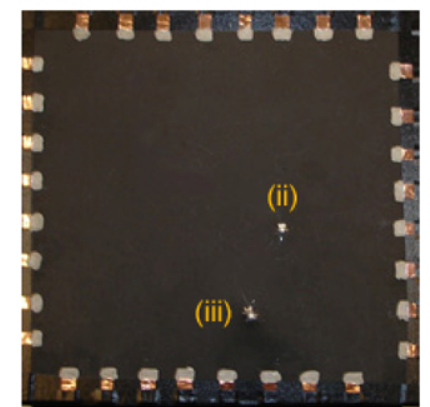

(a)

\section{BACK:}

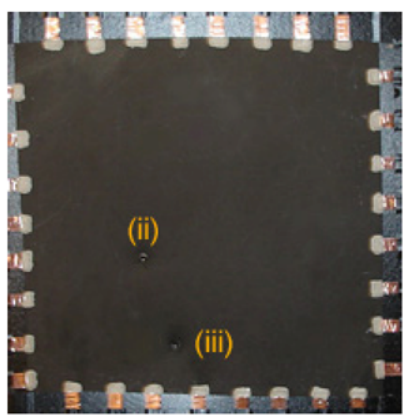

(d)

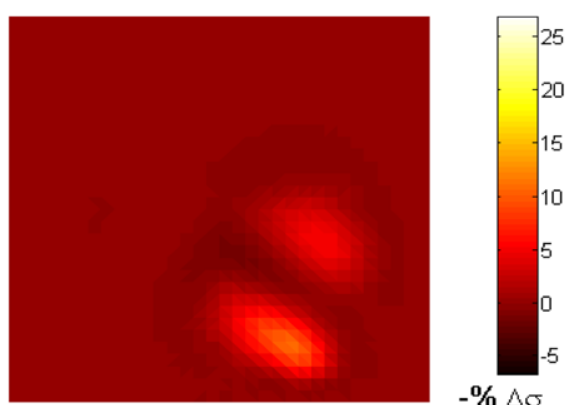

(b)

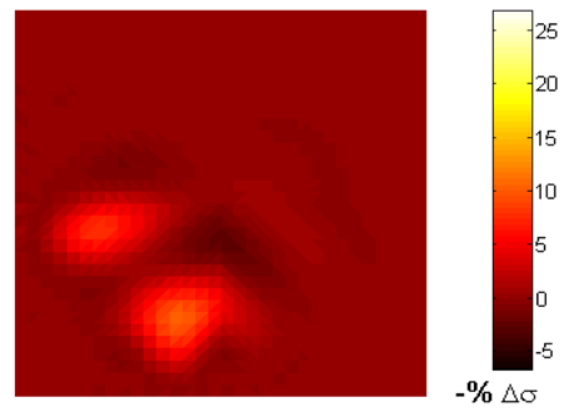

(e)

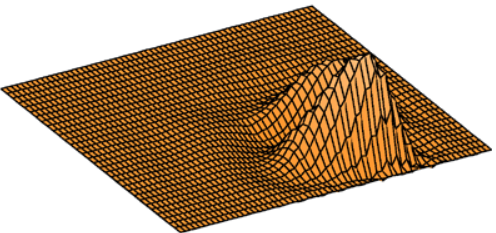

(c)

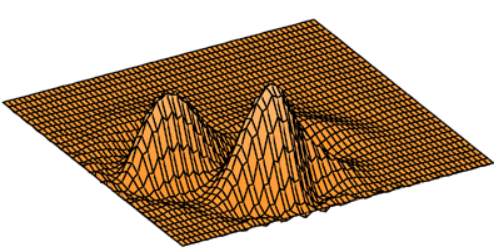

(f)
Fig. 11 Specimen IM-02 (first test): Two different magnitudes ([ii] $60^{\circ}$ and [iii] $90^{\circ}$ ) of pendulum hits are conducted. From the (a) front of the plate, the corresponding (b) 2D and (c) 3D EIT conductivity maps identify changes in film conductivity due to impact; the same is observed for the back side of the plate from the (d) photograph, (e) 2D, and (f) 3D EIT images 
Front:

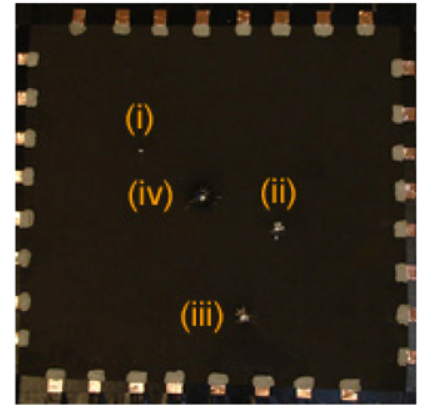

(a)

\section{BACK:}

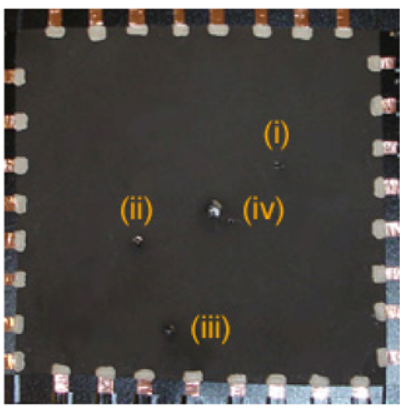

(d)

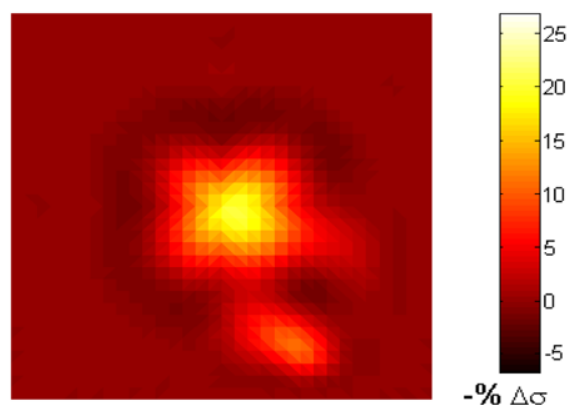

(b)

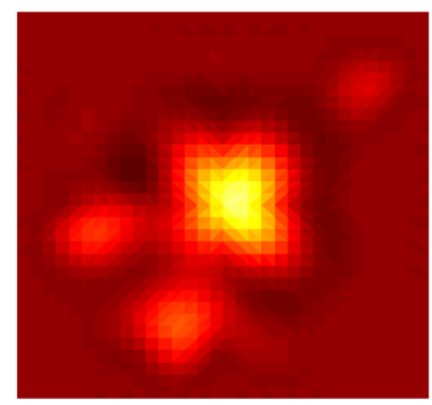

(e)

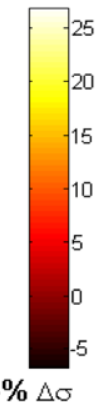

$-\% \Delta \sigma$

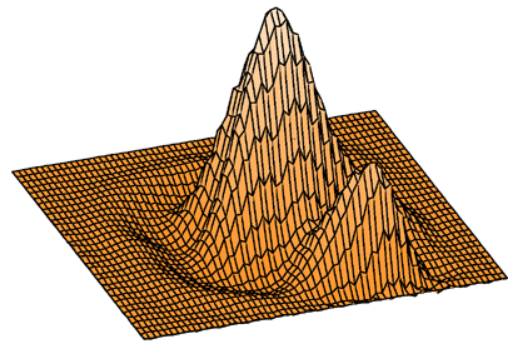

(c)

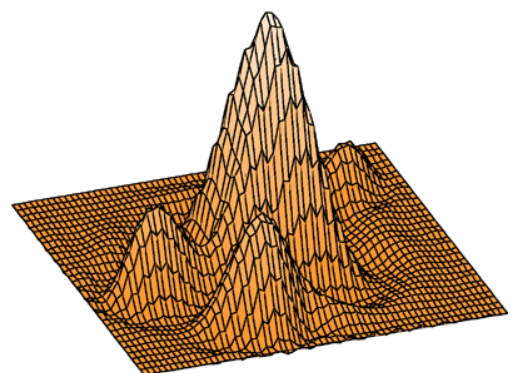

(f)
Fig. 12 Specimen IM-02 (second test): The same plate from Fig. 11 is impacted another two more times ([i] $30^{\circ}$ and [iv] $180^{\circ}$ ) at the top-right corner and middle of the plate as seen from the (a) front and (d) back side photographs. The (b) front-side 2D and (c) 3D EIT images, as well as the (e) back-side 2D and (f) 3D EIT conductivity maps, all verify that the proposed sensing skins are capable of detecting different magnitudes of induced damage and their corresponding locations ditional controlled impacts ([i] and [iv] shown in Figs. 12a and 12d) are conducted; the location and initial impact energies are tabulated in Table 2. It should be noted that the pendulum striker penetrated the entire plate at impact-[iv] (i.e., the most severe case of damage). Then, the EIT inverse problem is executed to obtain the 2D (Figs. 12b and 12e) and 3D (Figs. 12c and 12f) EIT spatial conductivity images. From Figs. 11 and 12, it can be concluded that, not only did the sensing skins capture new damage sustained on the aluminum alloy plates, but also, the EIT images also preserve damage which occurred 14 days earlier. Finally, when the average conductivity at each impact location is determined and plotted against initial impact energy, the response is near-linear as expected (Fig. 13). Thus, successful identification of the location and severity of impact damage on metallic structures (e.g., aircrafts, space vessels, ships, among others) permits timely repairs to prevent further damage from occurring. For instance, since small dents can serve as stress concentration points and facilitate crack growth, early detection and repair prolongs the structure's service lifetime and improves their reliability.

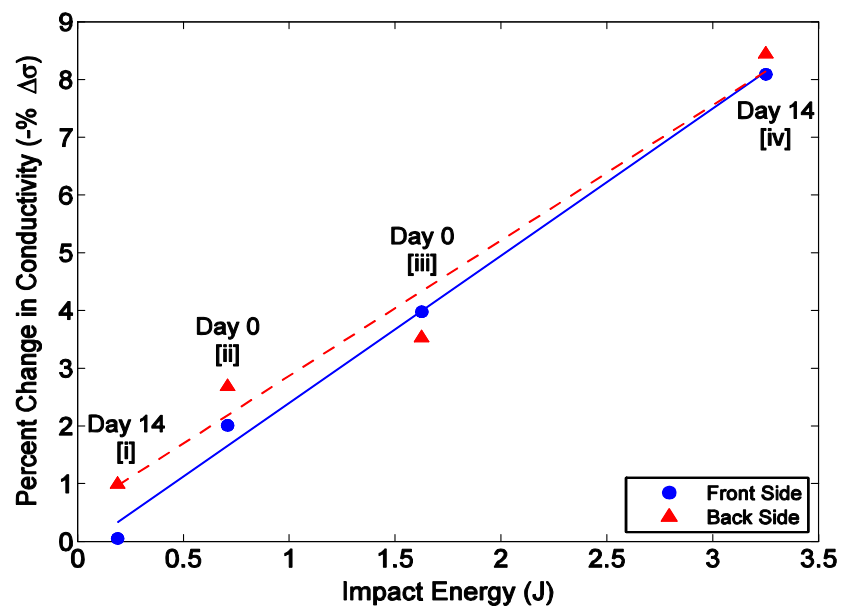

Fig. 13 The negative percent change in average film conductivity of specimen IM-02 increases near-linearly with increasing impact energy (as controlled by the initial potential energy of the pendulum impact testing apparatus). Times of inflicted impact damage are also denoted with each data point

\section{Conclusions}

In this study, a versatile layer-by-layer thin film assembly technique is employed for multifunctional nanocompos- 
ite fabrication. Previous studies have confirmed that these carbon nanotube-polyelectrolyte multilayer composites can be tailored with specific electromechanical and electrochemical sensing transduction mechanisms for strain and $\mathrm{pH} /$ corrosion monitoring. Physically, these thin films possess a highly percolated homogeneous structure, with deposition of individual or small bundles of single-walled carbon nanotubes within a compliant polymeric matrix $[16,17]$. In addition, these nanocomposites exhibit enhanced strength, stiffness, and ductility, such that ultimate film failure occurs beyond $10,000 \mu \varepsilon$ [27].

When combined with an electrical impedance tomographical (EIT) spatial conductivity mapping technique, these "sensing skins" have been validated for distributed strain and damage sensing. As opposed to a denselydistributed sensor instrumentation strategy, sensing skins can be coated onto structural surfaces to monitor distributed damage processes. Furthermore, instead of probing every discrete sensor for structural response data, EIT relies solely on boundary electrical measurements to reconstruct the 2D spatial conductivity distribution. Since skin electrical properties have been calibrated for specific applied external stimuli, changes in spatial film conductivity are directly correlated to deformation and damage.

First, (SWNT-PSS/PVA) 50 films are affixed onto PVC coupons for strain sensing. A tensile-compressive cyclic load pattern to $\pm 2,000 \mu \varepsilon$ is applied to specimens, and the load frame is paused at $1,000 \mu \varepsilon$ increments for EIT data acquisition. Results obtained confirm the skin's ability to detect strain during applied load. When the average conductivity change is computed at each applied strain, the results confirm the skin's linearity, stability, and high sensitivity $\left(S_{S}=6.5\right)$. It should be reiterated that this strain sensing validation is conducted with applied uniaxial stresses and strains. Since the nanocomposite morphology is based on a homogeneous percolated network of intertwined SWNTs, and that strain-induced electrical variations are a consequence of nanotube rigid-body movements within a compliant polymeric matrix, the proposed strain sensor only detects average strain (i.e., the magnitude of strain) at any given location. Similarly, changes in film conductivity does not distinguish between the directionality of strains (e.g., during biaxial loads) but rather outputs an averaged conductivity change. On the other hand, in order to enable identification of the directionality of strain, carbon nanotubes can be aligned within the polymeric matrix such that the sensor is insensitive to deformations occurring perpendicular to the aligned axis. Using the layer-by-layer technique, two perpendicularly aligned sensing layers can be fabricated within one multilayer thin film structure.

Upon strain sensing validation, the study is extended to utilize sensing skins for spatial impact damage detection.
The LbL technique is employed to deposit carbon nanotubebased composites onto large $\left(110 \times 110 \mathrm{~mm}^{2}\right)$ aluminum alloy substrates. Using a pendulum impact testing apparatus, controlled impacts are delivered onto sensing skin-coated aluminum specimens. Experimental results confirm that the skins are capable of identifying distributed impact damage location and severity. In addition, the impact damage sensing performance does not degrade over time (i.e., damage occurred over time is adequately detected via successive EIT measurements over a 14 day period), suggesting potential for long-term structural health monitoring.

In the near future, these skins will be validated for damage detection on actual structural components to illustrate their potential for more specific damage scenarios. For example, current work is focused on skin coatings to detect fatigue cracks in a structure. Moreover, long-term sensor stability tests will be conducted; here, skins will be exposed to ambient conditions to identify potential environmental factors that may affect sensor performance. In particular, outdoor use will be explored with skins exposed to weather conditions, ultraviolet (UV) sun light, among other factors. The layer-by-layer skin fabrication technique will also be aerosolized to facilitate efficient application onto existing structural elements such as gusset plates, steel girders, airfoils, among many other structural surfaces. Finally, work is underway in looking at the possibility of encoding electrochemical responses in nanoengineered thin films. For example, skins that change conductivity due to $\mathrm{pH}$ or ion-uptake could be used for distributed corrosion sensing.

Acknowledgements This research is supported by the National Science Foundation under grant CMMI-0528867 (Program Manager: Dr. S.C. Liu). Additional support is also provided by the Michigan Space Grant Consortium (MSGC) and National Aeronautics and Space Administration (NASA) and SPIE. In addition, the authors would like to express their gratitude towards Professor Victor Li and the ACE-MRL group for providing access to the MTS-810 load frame used during the experimental phase of this study.

\section{References}

1. Samsonov, P.: Nondestructive inspection of aging aircraft. Proc. SPIE 2001, 257-261 (1993)

2. Oberg, J.: The shuttle puzzle [Space Shuttle Columbia disaster]. IEEE Spectrum 40(3), 22-24 (2003)

3. Njord, J.R., Meyer, M.D.: Critical issues in transportation. Transp. Res. Board Natl. Acad. 1-13 (2006)

4. Doebling, S.W., Farrar, C.R., Prime, M.B.: Summary review of vibration-based damage identification methods. Shock Vib. Dig. 30(2), 91-105 (1998)

5. Raghavan, A., Cesnik, C.E.S.: Review of guided-wave structural health monitoring. Shock Vib. Dig. 39(2), 91-114 (2007)

6. Giurgiutiu, V., Cuc, A.: Embedded non-destructive evaluation for structural health monitoring, damage detection, and failure prevention. Shock Vib. Dig. 37(2), 83-105 (2005)

7. Giurgiutiu, V., Zagrai, A., Bao, J.: Damage identification in aging aircraft structures with piezoelectric wafer active sensors. J. Intell. Mater. Syst. Struct. 15(9-10), 673-687 (2004) 
8. Park, S., Yun, C.-B., Roh, Y., Lee, J.-J.: PZT-based active damage detection techniques for steel bridge components. Smart Mater. Struct. 15(4), 957-966 (2006)

9. Sohn, H., Park, G., Wait, J.R., Limback, N.P., Farrar, C.R.: Wavelet-based active sensing for delamination detection in composite structures. Smart Mater. Struct. 13(1), 153-160 (2004)

10. Gogotsi, Y.: (ed.): Nanomaterials Handbook. Taylor \& Francis, Boca Raton (2006)

11. Iijima, S.: Helical microtubules of graphitic carbon. Nature 354(6348), 56-58 (1991)

12. Saito, R., Dresselhaus, G., Dresselhaus, M.S.: Physical Properties of Carbon Nanotubes. Imperial College Press, London (1998)

13. Blighe, F.M., Lyons, P.E., De, S., Blau, W.J., Coleman, J.N.: On the factors controlling the mechanical properties of nanotube films. Carbon 46(1), 41-47 (2008)

14. Malik, S., Rosner, H., Hennrich, F., Bottcher, A., Kappes, M.M., Beck, T., Auhorn, M.: Failure mechanism of free standing singlewalled carbon nanotube thin films under tensile load. Phys. Chem. Chem. Phys. 6(13), 3540-3544 (2004)

15. Mamedov, A.A., Kotov, N.A., Prato, M., Guldi, D.M., Wicksted, J.P., Hirsch, A.: Molecular design of strong single-wall carbon nanotube/polyelectrolyte multilayer composites. Nat. Mater. 1(3), 190-194 (2002)

16. Loh, K.J., Kim, J., Lynch, J.P., Kam, N.W.S., Kotov, N.A.: Multifunctional layer-by-layer carbon nanotube-polyelectrolyte thin films for strain and corrosion sensing. Smart Mater. Struct. 16(2), 429-438 (2007)

17. Loh, K.J., Lynch, J.P., Shim, B.S., Kotov, N.A.: Tailoring piezoresistive sensitivity of multilayer carbon nanotube composite strain sensors. J. Intell. Mater. Syst. Struct. 19(7), 747-764 (2008)

18. Brown, B.H.: Electrical impedance tomography (EIT): a review. J. Med. Eng. Technol. 27(3), 97-108 (2003)
19. Holder, D.S. (ed.): Electrical Impedance Tomography: Methods, History and Applications. The Institute of Physics, London (2005)

20. Hou, T.-C., Loh, K.J., Lynch, J.P.: Spatial conductivity mapping of carbon nanotube composite thin films by electrical impedance tomography for sensing applications. Nanotechnology 18(31), 315501/315501-315501/315509 (2007)

21. Vauhkonen, M.: Electrical impedance tomography and prior information. Ph.D. Thesis, Kuopio University, Natural and Environmental Sciences, Kuopio, Finland (1997)

22. Wolfson, R., Pasachoff, J.M.: Physics with Modern Physics for Scientists and Engineers, 2nd edn. HarperCollins College, New York (1995)

23. Borcea, L.: Electrical impedance tomography. Inverse Probl. 18(6), R99-R136 (2002)

24. Cook, R.D., Malkus, D.S., Plesha, M.E., Witt, R.J.: Concept and Applications of Finite Element Analysis, 4th edn. Wiley, New York (2002)

25. Somersalo, E., Cheney, M., Isaacson, D.: Existence and uniqueness for electrode models for electric current computed tomography. SIAM J. Appl. Math. 52(4), 1023-1040 (1992)

26. Decher, G., Schlenoff, J.B. (eds.): Multilayer Thin Films: Sequential Assembly of Nanocomposite Materials. Wiley-VCH, Weinheim (2003)

27. Loh, K.J.: Development of multifunctional carbon nanotube nanocomposite sensors for structural health monitoring. Ph.D. Thesis, University of Michigan, Department of Civil and Environmental Engineering, Ann Arbor, MI (2008)

28. Decher, G.: Fuzzy nanoassemblies: toward layered polymeric multicomposites. Science 277(29), 1232-1237 (1997)

29. Moore, V.C., Strano, M.S., Haroz, E.H., Hauge, R.H., Smalley, R.E.: Individually suspended single-walled carbon nanotubes in various surfactants. Nano Lett. 3(10), 1379-1382 (2003) 